\title{
Inferior Removal of Dislocated Polymethyl Methacrylate Intraocular Lens and Scleral Refixation in Glaucomatous Eyes
}

\author{
Akihiko Shiraki · Susumu Sakimoto (D) · Yoshinori Oie · \\ Takeshi Soma $\cdot$ Atsuya Miki · Shinichi Usui · Shigeru Sato · \\ Kenji Matsushita $\cdot$ Hirokazu Sakaguchi $\cdot$ Kohji Nishida
}

Received: December 16, 2021 / Accepted: February 1, 2022 / Published online: February 21, 2022

(C) The Author(s) 2022

\section{ABSTRACT}

Introduction: The aim of this study was to report a technique for the removal of dislocated polymethyl methacrylate (PMMA) intraocular lenses (IOLs) in glaucomatous eyes.

Methods: Dislocated PMMA IOLs were removed from the inferior sclerocorneal incision, and sutureless intrascleral fixation of each IOL was performed to preserve the intact superior

Supplementary Information The online version contains supplementary material available at https:// doi.org/10.1007/s40123-022-00477-z.

A. Shiraki · S. Sakimoto $(\varangle) \cdot$ Y. Oie · T. Soma ·

A. Miki $\cdot$ S. Usui $\cdot$ S. Sato $\cdot$ K. Matsushita .

K. Nishida

Department of Ophthalmology, Osaka University

Graduate School of Medicine, 2-2 E7 Yamadaoka,

Suita, Japan

e-mail: susumu.sakimoto@ophthal.med.osaka-

u.ac.jp

S. Sakimoto $\cdot$ K. Nishida

Integrated Frontier Research for Medical Science

Division, Institute for Open and Transdisciplinary

Research Initiatives (OTRI), Osaka University, Suita, Japan

A. Miki

Department of Innovative Visual Science, Osaka

University Graduate School of Medicine,

Yamadaoka, Suita, Japan

H. Sakaguchi

Department of Ophthalmology, Gifu University

Graduate School of Medicine, Gifu, Japan conjunctiva and sclera for future trabeculectomy or to maintain a functional filtering bleb of trabeculectomy.

Results: In two cases, the condition of the bleb did not change, while the intraocular pressure improved or did not change after the procedures. IOL fixation was stable with no complications, such as tilt, decentration, or extrication of the IOL haptics.

Conclusion: This procedure of preserving the superior conjunctiva and sclera can maintain the function of the bleb, superior cornea and sclera and may contribute to the success of future trabeculectomy.

Keywords: Dislocated intraocular lens; Filtering bleb; Glaucoma; Polymethyl methacrylate; Sutureless intrascleral fixation 


\section{Key Summary Points}

Why carry out this study?

A treatment strategy for dislocated polymethyl methacrylate (PMMA) intraocular lens (IOL) removal and new intrascleral fixation in glaucomatous eyes have not yet been reported.

What was learned from the study?

Inferior removal of the dislocated PMMA IOL is a safe method to filter blebs in glaucoma.

This technique can reduce damage to the cornea and create a minimally invasive corneal incision.

This technique can preserve the superior conjunctiva for possible future filtering surgeries and maintain a good IOL fixation after trabeculectomy.

\section{DIGITAL FEATURES}

This article is published with digital features, including a video, to facilitate understanding of the article. To view digital features for this article go to: https://doi.org/10.6084/m9. figshare.19103558.

\section{INTRODUCTION}

The frequency of dislocation of an intraocular lens (IOL), a major postoperative complication after cataract surgery, has been reported to be $0.4-0.8 \%$ [1-3]. Pseudoexfoliation (PE) and uveitis are common risk factors. [4-6] However, eyes with PE or uveitis can be complicated by advanced secondary glaucoma that requires subsequent trabeculectomy $[7,8]$. Thus, in these cases, surgical technique for the repair of a dislocated IOL that can preserve the intact superior conjunctiva is needed for possible future trabeculectomy. However, if the dislocated IOL is a

polymethyl methacrylate (PMMA) IOL, an incision with a large width of $6 \mathrm{~mm}$ is required to remove it. To the best of our knowledge, a treatment strategy for dislocated PMMA IOL removal and new intrascleral fixation that minimizes superior conjunctival damage in glaucomatous eyes has not yet been reported. We report here the removal of a dislocated PMMA IOL from the inferior incision in eyes with functional or non-functional blebs of trabeculectomy. The surgical technique did not cause filtering bleb failure or a postoperative intraocular pressure (IOP) spike.

This study was performed in accordance with the Declaration of Helsinki, and all patients included in the study provided informed consent.

\section{METHODS}

Video 1 After incision of the 8 o'clock conjunctiva and sclerocorneal incision, the PMMA IOL was removed. Remaining Soemmering tissue was washed away while pushing the sclerocorneal incision site under perfusion flow. Since vitrectomy was performed, a new IOL was inserted through the 8 o'clock schlerocorneal incision. The inserted IOL was fixed in the sclera using the Yamane's method (MP4 $156725 \mathrm{~kb}$ )

The details of the proposed methodology are presented in Video 1. Initially, three ports for pars plana vitrectomy were set up with twin chandeliers (DORC Dutch Ophthalmic Research Center International BV, Zuidland, the Netherlands). After we made an incision of the inferior conjunctiva for the sclerocorneal incision, we created a $2.2-\mathrm{mm}$ superior corneal main port and two side ports, as is standard procedure for cataract surgery. We then moved the dislocated IOL onto the iris with a hook or anterior capsular forceps, following which a 6-mm inferior sclerocorneal incision was created with a $2.2-\mathrm{mm}$ slit knife (MANI Inc., Utsunomiya, Tochigi, Japan) at the 7-8 o'clock position (Fig. 1a). Then, the PMMA IOL was removed entirely from the $6-\mathrm{mm}$ inferior sclerocorneal incision (Fig. 1b). If any Soemmering tissue was left in the anterior chamber, it was washed away while pushing the sclerocorneal incision site under the perfusion flow of the IOP control system of the vitrectomy machine (Constellation Vitrectomy System; Alcon Laboratories, Inc., Fort Worth, TX, USA). After the incision was sutured and shortened 

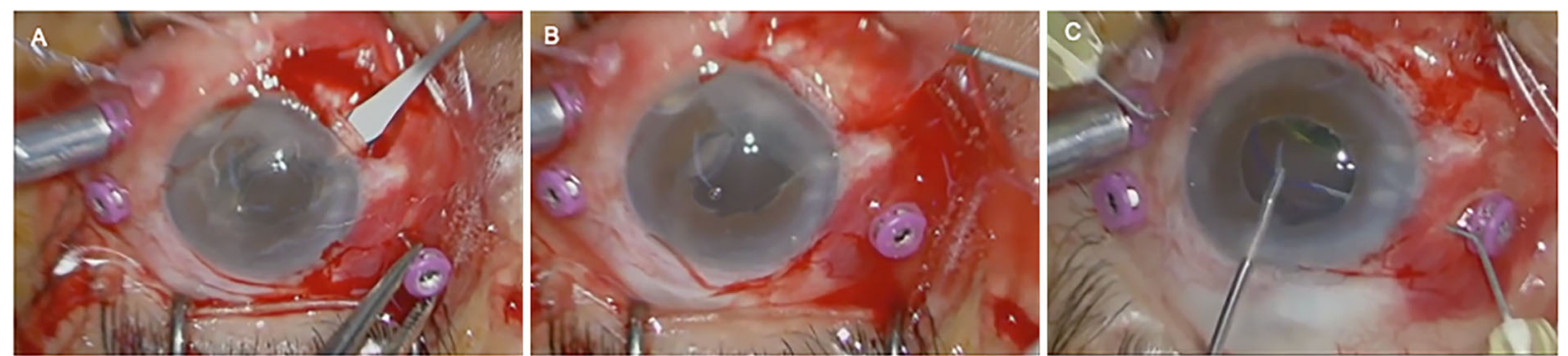

Fig. 1 Procedure for the removal of a PMMA IOL at the inferior schlerocorneal incision in the left eye of the first case (surgeon's view). a A limbal incision is created in the 8 o'clock position after a $6-\mathrm{mm}$ schlerocorneal incision is created in the inferior conjunctiva. b Removal of the dislocated PMMA IOL from the nasal-inferior schlerocorneal incision. c Sutureless fixation of IOL with the Yamane method. PMMA IOL Dislocated polymethyl methacrylate intraocular lens

fixation was stable with no complications, such as tilt, decentration or extrication of the IOL haptics (Fig. 2b, d). In the first case, postoperative astigmatism increased. The change in refraction may have been caused by damage to the corneal endothelium, resulting in a worsening of BK because the corneal endothelium with $\mathrm{BK}$ is thinner than that of normal eyes. However, this patient underwent DSAEK, which is originally scheduled after surgery for a dislocated IOL. Postoperative visual acuity (VA) in the second case did not change from the preoperative VA.

\section{DISCUSSION}

The presence of a filtering bleb in the superior conjunctiva prevents the surgeon from removing the dislocated IOL at the superior sclerocorneal incision. In nonfunctioning filtering blebs of trabeculectomy, the sclera and conjunctiva are vulnerable and are not suitable for creating an incision for IOL exchange. In contrast, in cases where the bleb is functioning, damage to the superior conjunctiva and sclera should be avoided. Therefore, removal of the dislocated IOL from different sites is necessary. With acrylic lenses, it is possible to cut and remove the dislocated IOL from the 3-mm corneal incision; however, with PMMA lenses, a corneal incision of at least 6-mm and multiple sutures of the cornea are required. Multiple sutures can cause astigmatism, which may lead to reduced VA. Therefore, it is important to 

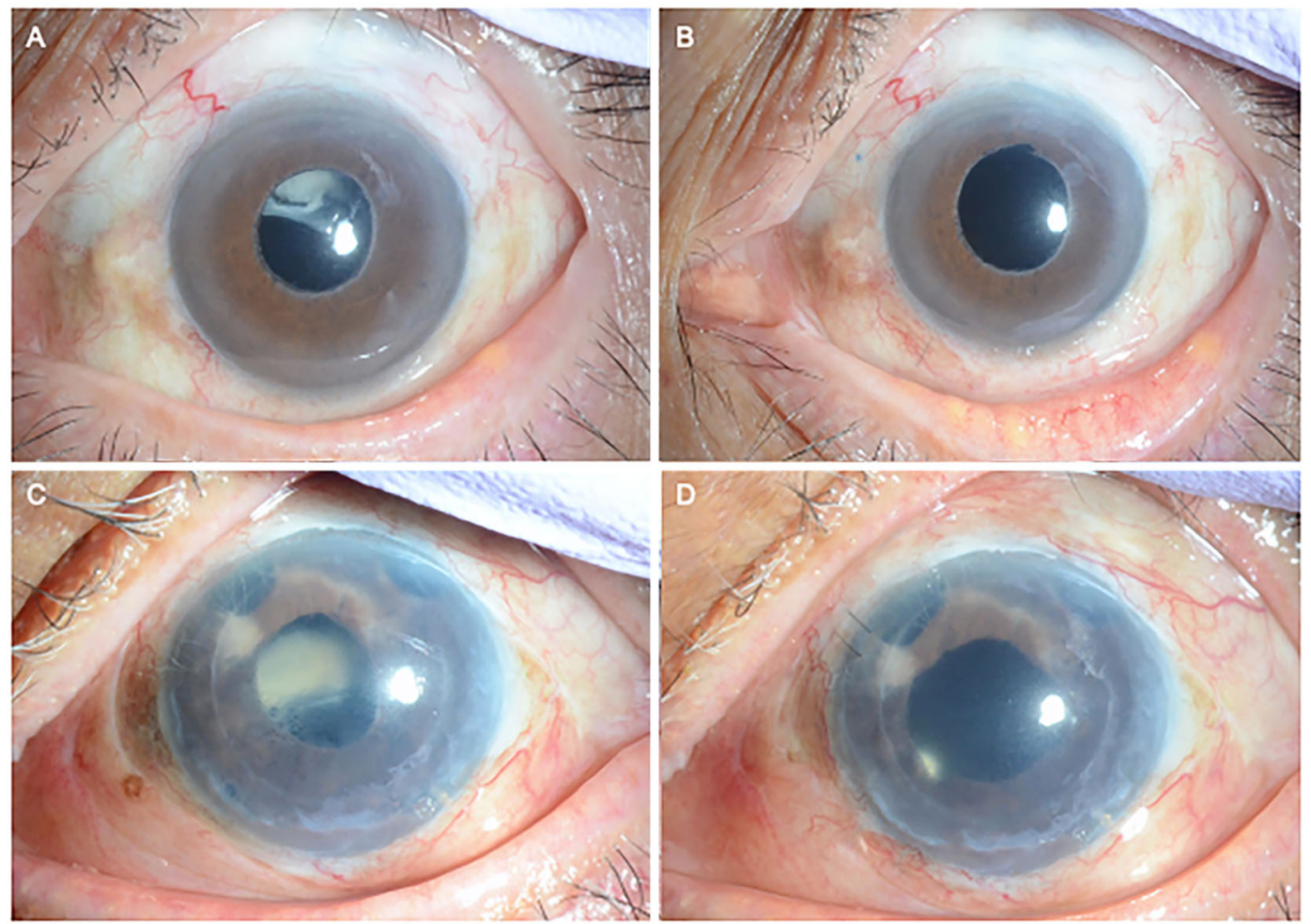

Fig. 2 Preoperative and postoperative anterior photographs. a The first case was a dislocated IOL with bullous keratopathy and diffuse bleb after trabeculectomy. b In the first case, the cornea and bleb did not change in the first 3 months postoperatively. c The second case

remove the PMMA IOL from the sclerocorneal incision and from locations other than the superior location. Mano et al. reported a case in which removal of a dislocated IOL and insertion of a new IOL were performed at the 8 o'clock position of the right eye during their combined surgery with trabeculectomy [10], with the aim of the inferotemporal approach for the removal and insertion of the right eye being the same, namely to preserve the intact superior conjunctiva and sclera. Similarly, our technique is also effective for preserving the superior conjunctiva and sclera, and especially effective for removing the dislocated PMMA IOL. Our technique has a number of advantages. First, the procedures of IOL removal and insertion and suturing at the inferior sclerocorneal incision are less invasive to the cornea; in particular, this technique appears to be useful in corneal-grafted eyes after penetrating keratoplasty and

preoperatively was a dislocated IOL with bullous keratopathy after DSAEK and three non-functional blebs after trabeculectomy. $\mathbf{d}$ In the second case, the cornea and bleb did not change 3 months postoperatively. DSAEK Descemet's stripping automated endothelial keratoplasty

DSAEK to avoid corneal damage that causes postoperative BK and delayed Descemet membrane folds. Second, this technique can protect the filtering bleb during trabeculectomy and preserve the intact superior conjunctiva for future trabeculectomy; this preservation is paramount to achieving successful trabeculectomy [11].

However, this technique has one important limitation: when a new acryl IOL is inserted with an IOL injector into the left eye, the patient's nose may limit the movement of the injector. There are two possible solutions to this problem. The first solution is to use a 2.2- to 2.4$\mathrm{mm}$ pre-placed superior incision of the cornea to insert the IOL, unless it is inserted after a corneal transplant. The small size of the 2.2- to 2.4-mm corneal incision reduces the risk of corneal damage without influencing the bleb of trabeculectomy. In the first case reported here, 
because the patient's nose was high, a new IOL was inserted through a superior corneal incision. However, in the second case, because the patient's nose was not high, it was possible to insert the IOL from below without any problems. The second solution is to alter the position of the sclerocorneal incision for IOL removal from the inferonasal incision to the inferotemporal incision in the left eye. If the incision for the removal of the dislocated IOL is created at the inferotemporal position, the positions of the three ports of vitrectomy must be changed, or the position of the infusion tube must be changed accordingly, from the inferotemporal port to the other port.

\section{CONCLUSION}

The inferior approach of dislocated IOL removal and new IOL insertion with a superior corneal main port and two side ports could become a standard technique that contributes to protecting the existing filtering bleb and leaving the intact superior conjunctiva for future trabeculectomy.

\section{ACKNOWLEDGEMENTS}

We would like to thank the participants for cooperating with this report.

Funding. No funding was received for this study and publication of this report. The journal's Rapid Service Fee was funded by the authors.

Authorship. All named authors meet the International Committee of Medical Journal Editors (ICMJE) criteria for authorship of this article, take responsibility for the integrity of the work as a whole, and have given their approval for this version to be published.

Author Contributions. Akihiko Shiraki and Susumu Sakimoto contributed to the concept, design and drafting of the manuscript. Yoshinori Oie, Takeshi Soma, Atsuya Miki, Shinichi Usui, Shigeru Sato and Kenji Matsushita drafted the manuscript. Hirokazu Sakaguchi and Kohji Nishida contributed to the concept and design.

Disclosures. Akihiko Shiraki, Susumu Sakimoto, Yoshinori Oie, Takeshi Soma, Atsuya Miki, Shinichi Usui, Shigeru Sato, Kenji Matsushita and Hirokazu Sakaguchi have nothing to disclose. Kohji Nishida received grant support from Alcon Laboratories, Inc. (Fort Worth, TX, USA).

Compliance with Ethics Guidelines. This study was performed in accordance with the Declaration of Helsinki and all patients included in the study provided informed consent.

Data Availability. Data sharing is not applicable to this article, as no datasets were generated or analyzed during the current study.

Open Access. This article is licensed under a Creative Commons Attribution-NonCommercial 4.0 International License, which permits any non-commercial use, sharing, adaptation, distribution and reproduction in any medium or format, as long as you give appropriate credit to the original author(s) and the source, provide a link to the Creative Commons licence, and indicate if changes were made. The images or other third party material in this article are included in the article's Creative Commons licence, unless indicated otherwise in a credit line to the material. If material is not included in the article's Creative Commons licence and your intended use is not permitted by statutory regulation or exceeds the permitted use, you will need to obtain permission directly from the copyright holder. To view a copy of this licence, visit http://creativecommons.org/licenses/by$\mathrm{nc} / 4.0 /$.

\section{REFERENCES}

1. Lee GI, Lim DH, Chi SA, Kim SW, Shin DW, Chung TY. Risk factors for intraocular lens dislocation after phacoemulsification: a nationwide populationbased cohort study. Am J Ophthalmol. 2020;214: 86-96. https://doi.org/10.1016/j.ajo.2020.03.012. 
2. Stark WJ, Worthen DM, Holladay JT, et al. The FDA report on intraocular lenses. Ophthalmology. 1983;90:311-7. https://doi.org/10.1016/s01616420(83)34555-3.

3. Kratz RP, Mazzocco TR, Davidson B, Colvard DM. The Shearing intraocular lens: a report of 1,000 cases. J Am Intra-Ocular Implant Soc. 1981;7:55-7.

4. Mönestam E. Frequency of intraocular lens dislocation and pseudophacodonesis, 20 years after cataract surgery-a prospective study. Am J Ophthalmol. 2019;198:215-22. https://doi.org/10. 1016/j.ajo.2018.10.020.

5. Jakobsson G, Zetterberg M, Lundström M, Stenevi U, Grenmark R, Sundelin K. Late dislocation of inthe-bag and out-of-the bag intraocular lenses: ocular and surgical characteristics and time to lens repositioning. J Cataract Refract Surg. 2010;36: 1637-44. https://doi.org/10.1016/j.jcrs.2010.04. 042 .

6. Gimbel HV, Condon GP, Kohnen T, Olson RJ, Halkiadakis I. Late in-the-bag intraocular lens dislocation: incidence, prevention, and management. J Cataract Refract Surg. 2005;31:2193-204. https:// doi.org/10.1016/j.jcrs.2005.06.053.
7. Panek WC, Holland GN, Lee DA, Christensen RE. Glaucoma in patients with uveitis. Br J Ophthalmol. 1990;74:223-7. https://doi.org/10.1136/bjo. 74.4.223.

8. Sayed MS, Lee RK. Recent advances in the surgical management of glaucoma in exfoliation syndrome. J Glaucoma. 2018;27(Suppl 1):S95-101. https://doi. org/10.1097/IJG.0000000000000918.

9. Yamane S, Sato S, Maruyama-Inoue M, Kadonosono K. Flanged intrascleral intraocular lens fixation with double-needle technique. Ophthalmology. 2017;124:1136-42. https://doi.org/10.1016/j. ophtha.2017.03.036.

10. Mano Y, Mizobuchi K, Watanabe T, Watanabe A, Nakano T. Minimally invasive surgery for intraocular lens removal and intrascleral intraocular lens fixation with trabeculectomy in a patient with dislocated intraocular lens and elevated intraocular pressure. Case Rep Ophthalmol. 2021;12:538-42. https://doi.org/10.1159/000511593.

11. Broadway DC, Grierson I, Hitchings RA. Local effects of previous conjunctival incisional surgery and the subsequent outcome of filtration surgery. Am J Ophthalmol. 1998;125:805-18. https://doi. org/10.1016/s0002-9394(98)00045-2. 